\title{
Promoting physical literacy in Irish adolescent youth: the youth-physical activity towards health (Y-PATH) intervention
}

\begin{abstract}
In their most contemporary model, strategic plan and call to action, the Aspen Institute have encouraged a cross-sector embrace towards the concept of 'physical literacy', specifically defined as the 'ability, confidence, and desire to be physically active for life.' This proposed physical literacy definition is a welcome addition to policy and practice for health, considering the unprecedented prevalence in recentyears of topical areas such as physical inactivity, movement inefficiency and unhealthy weight gain during childhood. Aligned with the United States primary objective of creating conditions for all youth to be physically literate by the middle schools years, the inception of the Youth-Physical Activity towards Health (Y-PATH) programme in Ireland is of particular consideration.

The Y-PATH school-based physical education (PE) intervention for adolescent youth was developed in 2011, as guided by the contextual Irish need for physical activity (PA) promotion and the subsequent wealth of literature surrounding this thematic field. In this evidence-based study, the reader will be introduced to the guiding principles of the intervention, specifically the educational focus of promoting physical literacy for adolescent youth.

The Y-PATH intervention consists of a multi-component whole-school approach to PA promotion in second level education. In terms of originality, the PE component of the intervention addresses psychosocial, health related activity (HRA) and fundamental movement skills (FMS) as particular strategies for increasing adolescent PA participation. All of the intervention components are grounded within a cost-efficient and feasible approach to overall physical literacy promotion.
\end{abstract}

Keywords: physical literacy, physical activity, physical education, fundamental movement skills, health related activity, adolescent, intervention
Volume 2 Issue 6 - 2015

\author{
Wesley O' Brien,' Sarahjane Belton, ${ }^{2}$ Johann \\ |ssartel ${ }^{3}$ \\ 'School of Education, Sports Studies and Physical Education \\ Department, University College Cork, Ireland

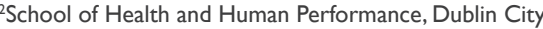 \\ University, Ireland
}

Correspondence: Hyacinth Wesley O Brien, Sports Studies and Physical Education Department, University College Cork, Ireland,Tel +3532149023 I 9, Email wesley.obrien@ucc.ie

Received: August 29, 2015 | Published: October 16,2015
Abbreviations: FMS, fundamental movement skills; HRA, health related activity; MRC, medical research council; PA, physical activity; PE, physical education; PEAI, physical education association of Ireland; QPE, quality physical education; UNESCO, united nations educational, scientific and cultural organization; YPAP, youth physical activity promotion

\section{Introduction}

In 2007, Margaret Whitehead outlined that the sporadic term of "physical literacy" was gaining momentum, specifically in relation to physical education (PE) practice in the United Kingdom. ${ }^{1}$ Most recently, in 2014, the Physical Education Association of Ireland (PEAI) launched their annual conference with the major focus towards "physical literacy" and the importance of building a "movement culture" during childhood. ${ }^{2}$ In the global context, physical literacy across the lifespan is now becoming a critical field of focus in physical activity (PA), exercise, sport settings and other public health sectors. ${ }^{3,4}$

Previous research, by Whitehead, contextualised physical literacy as a multifaceted conceptualisation of the skills required to fully realise physical activity potential through the embodied experience. ${ }^{5}$ In a more recent classification, Whitehead defined physical literacy as having the motivation, confidence, physical competence, knowledge and understanding that underpin someone's values and responsibilities for life-long purposeful physical pursuits. ${ }^{6}$ An important component of lifelong physical literacy development is the acquisition of fundamental movement skills (FMS). ${ }^{7}$

In most recentyears, the implementation of FMS programmes in sport, exercise and school-based environments has received considerable evidence-based attention. ${ }^{8-12}$ FMS as aligned with physical literacy, can be defined as basic observable patterns of behaviour and movement present from childhood to adulthood. ${ }^{13,14}$ The skills include, for example, running, hopping, skipping (locomotor), balancing, twisting, dodging (stability), and throwing, catching and kicking (object control). Individuals at the fundamental movement stage are preparing for the acquisition of more advanced skills within the sport specific stage. ${ }^{13,14}$ Whilst FMS are often considered the initial building blocks of more complex movements ${ }^{14}$ their specific mastery are a prerequisite for everyday movements, and participation in sports and PA. ${ }^{15,16}$

While physical literacy, as a concept, is the "new kid on the block", ${ }^{17}$ it is reasonable to state that both PA and physical fitness in childhood have been extensively researched over the past number of years. ${ }^{18-23}$ Undeniably, there is now a plethora of strong research evidence demonstrating that the physical fitness and health status 
of youth are substantially enhanced by regular PA participation. ${ }^{24-26}$ Lifestyle changes to people in industrialised countries over the past few years, has resulted in the decline of people engaging within physically active behaviours. ${ }^{27}$ A growing body of literature is now showing that the prevalence of chronic disease risk factors are increasing during adolescence, ${ }^{28,29}$ and that levels of PA decline dramatically during adolescence. ${ }^{30,31}$ Therefore, it is a valuable contribution to the literature that Tremblay \& Llyod's concept of physical literacy for youth specifies the importance of integrating assessment for FMS, PA and physical fitness. ${ }^{17}$ Yet, it must be recognised that knowledge of health is a critical component for skill development, PA participation and physical fitness; it is the foundation of characteristics, attributes, behaviours, awareness, and understanding related to healthy active living. ${ }^{17}$

Previous work by Alexanderon the alteration of an individual's knowledge base describes learning as a relatively permanent change in the way a person thinks and processes information. ${ }^{32}$ In light of the concept of physical literacy, it is interesting that research by Ennis suggests that participants can learn the kinaesthetic principles of fitness-related sciences within an educational fitness curriculum. ${ }^{33}$ The importance of knowledge within physical literacy ${ }^{17}$ is reinforced by Physical and Health Education Canada, specifically that PE programmes for youth provide the best opportunity to experience a variety of activities in a progressive, sequential format to ensure maximum learning and enjoyment. ${ }^{34}$ The "knowledge, skills and understanding" of physical literacy ${ }^{35}$ can be acquired constructively through the medium of PE for children and youth. ${ }^{36}$ In this particular instance, the provision of a knowledge based PE programme may provide a platform for the development of physically literate and physically active youth. ${ }^{8}$

Most recently, the concept of physical literacy across the lifespan has been subject to strategic action planning within the United States. ${ }^{4}$ Critical philosophical debate around physical literacy began in the mid-1990s, yet, there is now a global emphasis towards the promotion of physical literacy through the enactment of multi-sectoral approaches, ${ }^{3-5,35}$ with education firmly positioned as a platform for delivery. The purpose of the present evidence-based study is to highlight that many of the well-established components associated with physical literacy promotion (such as FMS, PA, health related knowledge and awareness, etc...) are currently being delivered in Irish second-level schools for adolescent youth, aged 12 to 15 years old, as part of the longitudinal Y-PATH randomised-controlled trial.

\section{Case presentation}

\section{Synopsis y-path intervention}

A systematic review on youth PA intervention effectiveness concluded that for adolescent youth, multi component interventions involving the school, family and community have the potential to make important differences in the increase of youth PA. ${ }^{37}$ More recently, a systematic review summarising the effectiveness of schoolbased interventions in promoting youth PA and fitness, found that the evidence continues to advocate for the on-going implementation of school-based PA interventions for youth. ${ }^{24}$ The Y-PATH programme is fundamentally guided by research informed findings. ${ }^{8,38}$ As previously reported in $\mathrm{O}^{\prime}$ Brien et al., ${ }^{39}$ there are four essential intervention components within the existing Y-PATH evidence-based study:

Student component: A targeted focus on the integration of HRA and FMS for students within a specifically tailored post-primary PE curriculum (delivered by specialist PE teachers).
Parent/guardian component: A PA promotion (across the lifespan) workshop prior to the beginning of the intervention, and distribution of research informed Y-PATH information leaflets for parents and guardians.

Teacher component: All school teachers attend two workshops (pre and mid intervention) which among other concepts, highlight the importance of "active role modelling", and voluntary participation in a one week "Teacher Pedometer Challenge" to enhance participation and compliance.

Website component: All student, parent and teacher resources are made readily available for all intervention participants (http://www. dcu.ie/shhp/y_path.shtml).

A comprehensive overview and theoretical structure for the Y-PATH programme has been reported elsewhere; ${ }^{8}$ (Figure 1) contextualises the component structure of the intervention.

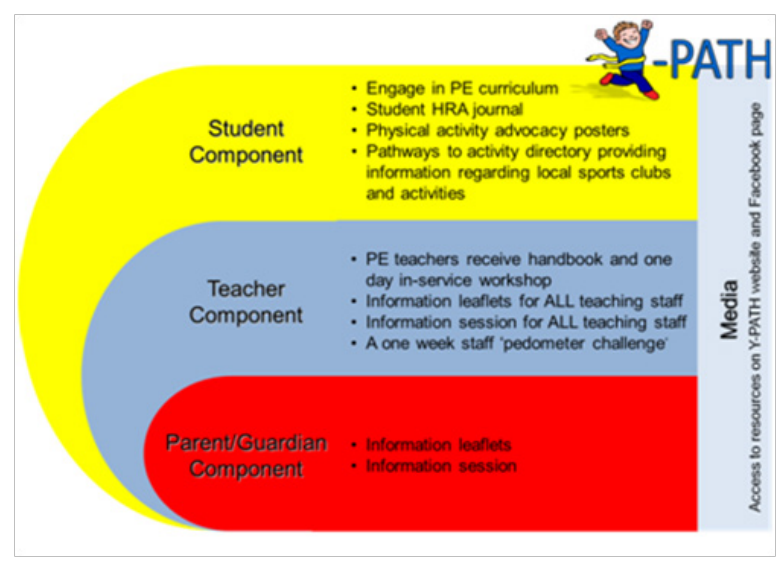

Figure I Overview of the structure of the Y-PATH programme. ${ }^{8}$

In line with the promotion of physical literacy, ${ }^{5,6,40}$ it is important to note that the Y-PATH intervention was developed with a strong focus on PE based HRA ${ }^{41-46}$ and FMS, ${ }^{47-50}$ with additional school, teacher and parental components.

\section{Research considerations in the development and extension of the $y$-path intervention}

This Y-PATH intervention was initially based on the Medical Research Council's (MRC) guidance document, 'Developing and Evaluating Complex Interventions', ${ }^{51}$ which is shown in Figure 2. Since the inception of this evidence-based study, the Y-PATH programme has progressed from the theory/modelling phase one, ${ }^{8,38}$ to the exploratory trial phase two $\mathrm{o}^{39}$ and is currently, undergoing the definitive randomised-controlled trial of phase three. The four stages, as outlined in the MRC document, for 'Developing and Evaluating Complex Interventions' are: 1) Development, 2) Feasibility, 3) Evaluation, and 4) Implementation.

Following the MRC's guidelines, ${ }^{51}$ the Y-PATH research study is continuing to generate and increase the longitudinal evidence-base. The development of the Y-PATH intervention (phase one theory/ modelling 2010-2011) was guided by previously reported research findings, ${ }^{8}$ specifically the low levels of PA participation and FMS proficiency amongst Irish adolescent youth. As part of this theory and modelling phase, the development of the Y-PATH intervention also identified an evidence-base of literature relating to youth PA and FMS promotion during PE classes. The development of this intervention then used the Youth Physical Activity Promotion (YPAP) model as a 
theoretical framework. ${ }^{52}$ The Y-PATH intervention hypothesised that if the research team (Principal Investigators and trained field staff) were successful in positively influencing the enabling, predisposing and reinforcing factors for PA experienced by youth, then a successful adolescent PA intervention would occur. The purpose of using the YPAP framework, as part of the theory/modelling phase, was to collect specific data, including levels of PA, FMS, body mass index and psychological influences (including attitudes and selfefficacy), so that a meaningful and relevant intervention could be implemented. ${ }^{8}$ Phase two's 'Exploratory Trial' (2011-2012) within the MRC framework evaluated the Y-PATH intervention effectiveness after nine and twelve months respectively. Findings from this quasiexperimental non-randomised controlled trial suggested a positive effect for the Y-PATH intervention in the increase of PA and FMS levels of Irish adolescent youth. ${ }^{39}$

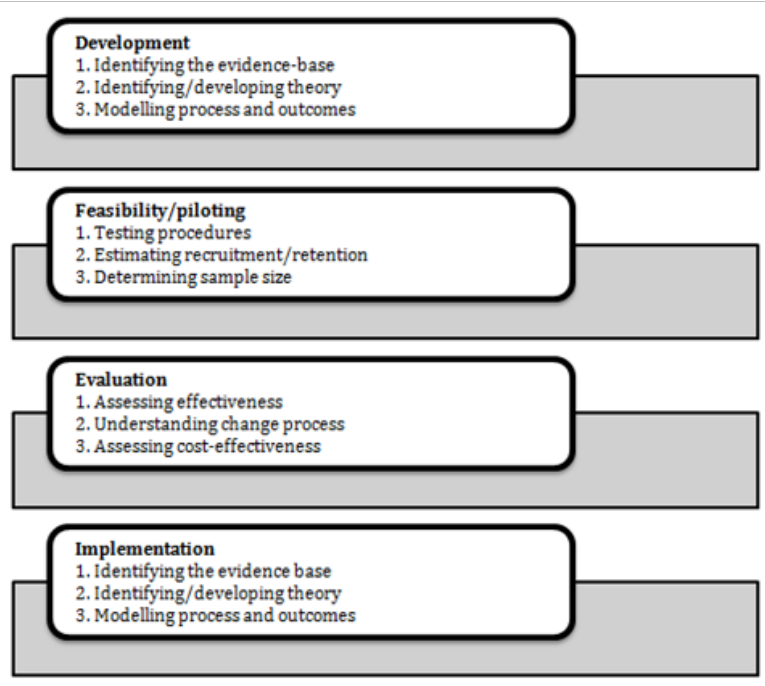

Figure 2 Key elements of the development and evaluation process. ${ }^{5}$

The Y-PATH intervention, ${ }^{8}$ was therefore, shown to be effective in increasing adolescent PA and FMS levels. As part of the definitive randomised controlled trial (phase three 2013-2014), the Y-PATH research team were looking at the evaluation of the programme's evidence base on a larger population sample (data analysis and results under review) to further demonstrate the impact of Y-PATH on PA and FMS levels.

Within the Y-PATH intervention, physical literacy promotion is ideally positioned for Irish post-primary school PE, specifically to foster students' development of the skills, knowledge, and attitudes needed to become physically literate..$^{53}$ Through this evidence-based Y-PATH programme, ${ }^{8,39}$ students become physically active and skilled through PE, which in turn enables them to demonstrate the core components of physical literacy throughout the lifespan. A positive spiral towards physical literacy promotion for adolescent youth can be achieved within the Y-PATH intervention, specifically with the emphasis on 'quality, well-delivered PE'. ${ }^{33}$

\section{Making the physical literacy case for the y-path programme in Ireland}

In her response to 'Physical Literacy in the context of Physical Education in the Secondary School', Whitehead outlined:

"The development of physical literacy depends as much, if not more, on the nature of the interaction between the teacher and the pupil, as on the content of the lesson. Above all physical education must provide a positive and rewarding experience for all young people-whatever their ability. At the heart of Physical Literacy is the motivation to take part in physical activity. This is acquired as young people make progress in movement mastery and develop selfconfidence and self-esteem in this significant aspect of their human potential. $^{54}$

From the recently published evidence, it appears that positive school-based PE provides an opportunistic window for physical literacy promotion across the lifespan, ${ }^{4-6,17,40}$ particularly for youth. In this section, Whitehead and Murdoch's conceptual map on the attainment and maintenance of lifelong physical literacy for second level youth ${ }^{55}$ will be documented specifically in relation to the Y-PATH programme (Table 1). ${ }^{56}$

Table I A comparative relationship between Whitehead and Murdoch's conceptual map and the Y-PATH programme in the attainment of physical literacy for secondary school youth

\section{Stages in attaining and maintaining physical literacy secondary school ${ }^{55}$}

Development of Physical Literacy as a fundamental goal of Physical Education. Fundamentals of Physical Literacy established and contextualised in a range of physical activities.

Activity opportunities outside of school introduced.

\section{Personnel influencing the attainment} and maintenance of Physical literacy in secondary school ${ }^{55}$

Teachers, parents, family, peers, coaches, club and local facility personnel.

\section{Stages in attaining and maintaining physical literacy in Y-PATH}

Development of PA, FMS, HRA and psychosocial health is the goal of Y-PATH Physical Education. Fundamentals of Physical Literacy and components of FMS contextualised in the 7 strands of Irish Junior Cycle Physical Education ${ }^{56}$ (adventure activities, aquatics, athletics, dance, games, gymnastics, HRA).

Activity opportunities outside of school introduced through 'Pathways to Activity' initiative as part of the Y-PATH intervention (inventory of and links to the extra-curricular opportunities for PA and sport within the local community).

\section{Personnel influencing the attainment and maintenance of physical literacy in Y-PATH}

Teachers (PE specialists and non-specialists) receive

Y-PATH leaflets attend PA workshops and engage in 'pedometer challenge'.

Parents/family receives Y-PATH leaflets and attend PA workshop.

Coaches, club and local facility personnel contact details (email, url, phone etc.) available in the 'Pathways to Activity' document. 
Table continued.

Situations, contexts where Physical Literacy can be encouraged, established and maintained in secondary school ${ }^{55}$

\section{Situations, contexts where Physical Literacy can be encouraged, established and} maintained in Y-PATH
School Physical Education, extracurricular opportunities. Sports/activity clubs. Home, local environment, local facilities.
Y-PATH is a multi-component whole-school approach to PA promotion, with school physical education the medium for programme dissemination. The developed resources also provide direct links to the local sports clubs, home, environment and facilities.

\section{Discussion}

In the present Y-PATH evidence-based study, the authors are creating awareness and equally advocating for an approach to increasing adolescent PA participation through the structured teaching of secondary school PE in Ireland, that is fundamentally rooted within components of physical literacy promotion. ${ }^{54}$ The domains of the Canadian Assessment of Physical Literacy include 'physical activity behaviours, physical fitness, motor skills, awareness, knowledge and understanding, $;{ }^{17}$ from the evidence-based study presented, it is clear that the Y-PATH programme is targeting the promotion of physically literate secondary school youth through the medium of PE, as delivered by specialist teachers. Whitehead has suggested that physically literate individuals ought to possess assurance and selfconfidence in parallel with their movement proficiency. ${ }^{5}$

In a recent publication from the United Nations Educational, Scientific and Cultural Organization (UNESCO) in 2015 on 'Quality Physical Education(QPE) guidelines for policy-makers', ${ }^{57}$ documented the importance of physical literacy for healthy, able and active citizens. From this policy guideline document, ${ }^{57}$ UNESCO outlined that QPE should comprise of the following:

1. QPE should enable children and young people to become physically literate, and provision should feature from the early years through the entire school journey to secondary school education.

2. Fundamental movement skills are a vital aspect of physical literacy and, also, to the development of healthy, able, and active citizens

3. The promotion of physical literacy should then remain a key feature of any physical education curriculum throughout primary and secondary education. ${ }^{57}$

From the presentation of this evidence-based study, physical literacy promotion is clearly a strong platform within the delivery of Y-PATH PE. The core components of physical literacy (PA behaviours, motor skills, physical fitness, PA knowledge, awareness, and understanding) have been at the heart of the Y-PATH programme since its inception in 2010. This was not due to a direct attempt of researchers to address physical literacy per say, but rather was driven by the research identified needs evident in Irish adolescent youth ${ }^{8}$ for whom the intervention was developed. The attainment of physically literate youth is readily integrated and embedded within Y-PATH, specifically as learners encounter a range of age and stage appropriate opportunities. ${ }^{57}$

\section{Conclusion}

Over the past decade, many public health agencies have introduced and embraced a variety of initiatives based on the desired outcome of physically literate individuals and populations. ${ }^{4}$ While this concept is primarily aimed at young people, physical literacy programmes seek to provide the motivation, confidence, physical competence, knowledge and understanding to be active for life. ${ }^{6}$ With this emergent shift towards physical literacy, the Y-PATH school-based PE intervention for Irish adolescent youth is well positioned for addressing this call to action. This Y-PATH evidence-based study has introduced the guiding principles of the PE-based intervention, specifically the sustainability of learning to be active during adolescence. It is important to note that the Y-PATH intervention consists of a multi-component approach to whole-school PA promotion, many components which reflect the integration of physical literacy in the school environment. By actively engaging the student, teacher, parent, guardian and local community in the intervention process, the Y-PATH intervention is adhering to Whitehead and Murdoch's previously published conceptual map in the attainment of lifelong physical literacy. ${ }^{55}$ In terms of originality, the PE components of the intervention addresses psychosocial, HRA and FMS content in the promotion of skill competency, attitudes, selfefficacy and educational belief towards the importance of sustainable PA participation. The intervention is grounded within a cost-efficient and feasible approach to overall physical literacy promotion in the school context. The interpretation of the physical literacy concept continues to be refined with updated research informed data ${ }^{3,4,6,11}$ and contributions to this 'new kid on the block' are set to continue. This evidence-based study set out to highlight how physical literacy is being promoted within a school-based programme for Irish adolescent youth.

\section{Acknowledgements}

Research for the Y-PATH programme was supported by Dublin City University (Ireland), the Local Sports Partnerships (LSPs) in Wicklow, Dublin (Dublin City, Dun Laoghaire-Rathdown, Fingal and South Dublin County) and the Wicklow Vocational Education Committee (VEC). These funding contributors had no input in study design, in the collection, analysis and interpretation of data, in the writing of this manuscript, or in the decision to submit the article for publication. We wish to acknowledge the trained field staff for their dedicated professionalism during each phase of data collection and analysis. Finally, a sincere gratitude to the participants, parents, guardians, teachers and principals from all of the schools involved in the Y-PATH programme to date.

\section{Conflict of interest}

The author declares no conflict of interest.

\section{Refererences}

1. Whitehead M. Physical literacy: Philosophical considerations in relation to developing a sense of self, universality and propositional knowledge. Sport, Ethics and Philosophy. 2007;1(3):281-298. 
2. Physical Education Association of Ireland. Physical education and physical literacy: Building a movement culture. Ireland: Physical Education Association of Ireland; 2014.

3. Giblin S, Collins D, Button C. Physical literacy: Importance, assessment and future directions. Sports Medicine. 2014;44(9):1177-1184.

4. The Aspen Institute. Physical literacy in the United States: A model, strategic plan, and call to action. 2015.

5. Whitehead ME. Physical literacy: Throughout the life course. London; 2010

6. Whitehead M. The definition of physical literacy. 2013.

7. Haywood KM, Getchell N. Life span motor development. 5th ed. USA: Human Kinetics Champaign; 2009.

8. Belton S, O Brien W, Meegan S, et al. Youth-Physical Activity Towards Health: Evidence and background to the development of the Y-PATH physical activity intervention for adolescents. BMC Public Health. 2014:14(122)

9. Bryant ES, Duncan MJ, Birch SL. Fundamental movement skills and weight status in British primary school children. Eur J Sport Sci. 2014;14(7):730-736.

10. Burrows EJ, Keats MR, Kolen AM. Contributions of after school programs to the development of fundamental movement skills in Children. Int $J$ of Exercise Sci. 2014;7(3):236-249.

11. Castelli DM, Centeio EE, Beighle AE, et al. Physical literacy and comprehensive school physical activity programs. Prev Med. 2014;66:95-100.

12. Lai SK, Costigan SA, Morgan PJ, et al Do school-based intervention focusing on physical activity, fitness, or fundamental movement skill competency produce a sustained impact in these outcomes in children and adolescents? A systematic review of follow-up studies. Sports Med. 2014;44(1):67-79.

13. Department of Education Victoria. Fundamental motor skills: A manual for classroom teachers. Melbourne, Australia; 1996.

14. Gallahue DL, Ozmun JC, Goodway JD. Understanding motor development: Infants, children, adolescents, adults. 7th ed. New York; 2012.

15. Lubans DR, Morgan PJ, Cliff DP, et al. Fundamental movement skills in children and adolescents: Review of associated health benefits. Sports Med. 2010;40(12):1019-1035.

16. Stodden DF, Goodway JD, Langendorfer SJ, et al. A developmental perspective on the role of motor skill competence in physical activity: An emergent relationship. Quest. 2008;60:290-306.

17. Tremblay M, Lloyd M. Physical literacy measurement - The missing piece. Physical and Health Education. 2010;76(1):1-5

18. American College of Sports Medicine. Opinion statement on physical fitness in children and youth. Med \& Sci in Sports \& Exe. 1988;20:422423.

19. Aibar A, Bois JE, Generelo E, et al. A cross-cultural study of adolescents' physical activity levels in France and Spain. Eur J Sport Sci. 2013;13(5):1-8.

20. Currie C, Zanotti C, Morgan A, et al. Social determinants of health and well-being among young people. Health Behaviour in School-aged Children (HBSC) study: International report from the 2009/2010 survey. Copenhagen; 2012.

21. Eaton DK, Kann L, Kinchen S, et al. Youth risk behavior surveillanceUnited States, 2011. Morbidity and Mortality Weekly Report. 2012;61(4):1-162.
22. Fransen J, Deprez D, Pion J, et al. Changes in physical fitness and sports participation among children with different levels of motor competence: A two-year longitudional study. Pediatric Exerc Sci. 2014;26(1):11-21.

23. Sacchetti R, Ceciliani A, Garulli A, et al. Physical fitness of primary school children in relation to overweight prevalence and physical activity habits J Sports Sci. 2012;30(7):633-640.

24. Dobbins M, Husson H, Decorby K, et al. School-based physical activity programs for promoting physical activity and fitness in children and adolescents aged 6 to 18 (Review). Cochrane Database of Syst Rev. $2013 ;(2): 1-260$.

25. Phyiscal Activity Guidelines Advisory Committee. DC: Department of Health and Human Services; 2008.

26. Rauner A, Mess F, Woll A. The relationship between physical activity, physical fitness and overweight in adolescents: A systematic review of studies published in or after 2000. BMC Pediatrics. 2013:13(19).

27. Bouchard C, Blair SN, Haskell WL. Physical activity and health Champaign, IL: Human Kinetics; 2007.

28. May AL, Kuklina EV, Yoon PW. Prevalence of cardiovascular disease risk factors among US adolescents, 1999-2008. Pediatrics. 2012;129(6):1035-1041.

29. Woods CB, Tannehill D, Quinlan A, et al. The children's sport participation and physical activity study (CSPPA). Research Report No 1. Ireland: Dublin; 2010

30. O’Donovan G, Blazevich AJ, Boreham C, et al. The ABC of physical activity for health: A consensus statement from the British association of sport and exercise sciences. J Sports Sci. 2010;28(6):573-591.

31. Ortega FB, Konstabel K, Pasquali E, et al. Objectively measured physical activity and sedentary time during childhood, adolescence and young adulthood: A cohort study. PloS One. 2013;8(4):1-8.

32. Alexander PA. Psychology in learning and instruction. Columbus USA; 2006.

33. Ennis CD. On their own: Preparing students for a lifetime. Journal of Physical Education, Recreation \& Dance. 2010;81(5):17-22.

34. Physical and Health Education Canada. What is the relationship between Physical Education and Physical Literacy? The Centre for Healthy Development, Brock University; 2009

35. Sport Northern Ireland. Developing Children's Physical Literacy. Belfast; 2009.

36. Hills AP, Dengel DR, Lubans DR. Supporting public health priorities: Recommendations for physical education and physical activity promotion in schools. Progress In Cardiovascular Diseases. 2015;57(4):368-374.

37. Van Sluijs EMF, McMinn AM, Griffin SJ. Effectiveness of interventions to promote physical activity in children and adolescents: Systematic review of controlled trials. British J Sports Med. 2007;42:653-657.

38. O Brien W, Belton S, Issartel J. Fundamental movement skill proficiency amongst adolescent youth. Physical Education and Sport Pedagogy, In Press; 2015.

39. O Brien W, Issartel J, Belton S. Evidence for the efficacy of the YouthPhysical Activity towards Health (Y-PATH) intervention. Advances in Physical Education. 2013;3(4):145-153.

40. Loitz C. The importance of lifelong physical literacy. Alberta Centre for Active Living - Research and Education for the Promotion of Physical Activity. Well Spring: Sharing Physical Activity Knowledge. 2013:24(4).

41. Gittelsohn J, Steckler A, Johnson CC, et al. Formative research in school and community-based health programs and studies:"State of the art" and the TAAG approach. Health Educ Behav. 2006;33(1):25-39. 
42. Le Masurier G, Corbin CB. Top 10 Reasons for quality physical education. Journal of Physical Education, Recreation and Dance. 2006;77(6):44-53.

43. McKenzie TL, Nader PR, Strikmiller PK, et al. School physical education: Effect of the child and adolescent trial for cardiovascular health. Preventive Medicine. 1996;25(4):423-431.

44. McKenzie TL, Sallis JF, Prochaska JJ, et al. Evaluation of a two-year middle-school physical education intervention: M-SPAN. Med Sci in Sports Exerc. 2004;36(8):1382-1388.

45. Murillo Pardo B, García Bengoechea E, Generelo Lanaspa E, t al. Promising school-based strategies and intervention guidelines to increase physical activity of adolescents. Health Educ Res. 2013;28(3):523-538.

46. Sallis JF, McKenzie TL, Beets MW, et al. Physical education's role in public health: Steps forward and backward over 20 years and HOPE for the future. Res Qfor Exerc Sport. 2012;83(2):125-135.

47. Kalaja SP, Jaakkola TT, Liukkonen JO, et al. Development of junior high school students' fundamental movement skills and physical activity in a naturalistic physical education setting. Physical Education and Sport Pedagogy. 2012;17(4):411-428.

48. Martin EH, Rudisill ME, Hastie PA. Motivational climate and fundamental motor skill performance in a naturalistic physical education setting. Physical Educ Sport Pedagogy. 2009;14(3):227-240.
49. Mitchell B, McLennan S, Latimer K, et al. Improvement of fundamental movement skills through support and mentorship of class room teachers. Obes Res Clin Pract. 2013;7(3):e230-e234.

50. Mostafavi R, Ziaee V, Akbari H, et al. The effects of SPARK physical education program on fundamental motor skills in 4-6 year-old children. Iran J Pediatrics. 2013;23(2):216-219.

51. Medical Research Council. Developing and evaluating complex intervention: New guidance. MRC Health Services and Public Health Research Board. 2008.

52. Welk G. The youth physical activity promotion model: A conceptual bridge between theory and practice. Quest. 1999;51(1):5-23.

53. Mandigo J, Francis N, Lodewyk K, et al. Position paper: Physical literacy for educators. Physical and Health Education Canada. 2009.

54. Killingbeck M, Bowler M, Golding D, et al. Physical education and physical literacy. Practice Matters. 2007:20-24.

55. Whitehead M, Murdoch E. Physical literacy and physical education: Conceptual mapping. PE Matters. 2006;1(1):6-9.

56. Department of Education and Skills. Junior cycle physical education. Dublin: The Stationary Office; 2003.

57. United Nations Educational, Scientific and Cultural Organization. Quality physical education: Guidelines for policy makers. Paris; 2015. 